\title{
DECOMPOSIÇÃO E LIBERAÇÃO DE NUTRIENTES DA SERAPILHEIRA FOLIAR EM POVOAMENTOS DE Eucalyptus grandis NO NORTE FLUMINENSE ${ }^{1}$
}

\author{
Gilmar Santos Costa ${ }^{2}$, Antônio Carlos da Gama-Rodrigues ${ }^{3}$, Gláucio de Melo Cunha ${ }^{4}$
}

\begin{abstract}
RESUMO - O objetivo deste trabalho foi avaliar a decomposição e liberação de nutrientes da serapilheira foliar em um plantio de Eucalyptus grandis de primeira rotação, com 8 anos, e em rebrotas com 2 e 5 anos de idade, por meio da técnica de sacos de serapilheira. A temperatura média anual foi de $19,5^{\circ} \mathrm{C}$ e a precipitação pluvial anual, de $850 \mathrm{~mm}$, com 54\% das chuvas concentradas no período de outubro a dezembro. Os resultados indicaram que foi similar o porcentual de perda de massa, bem como a liberação de frações orgânicas (lignina, celulose e polifenol) e de nutrientes ( $\mathrm{N}, \mathrm{P}, \mathrm{K}, \mathrm{Ca}$ e $\mathrm{Mg}$ ) da serapilheira foliar para o plantio de primeira rotação e as rebrotas. O porcentual de perda de massa, média dos três povoamentos, ajustou-se a um modelo quadrático $\left(y=99,443+0,0348 x-0,0003 x^{2} ; R^{2}=0,92 ; p=0,0225\right)$. Somente no período de maiores precipitações observouse a efetiva perda de massa (29-33\%), assim como a liberação de celulose (40 - 51\%), de polifenóis (54 - 70\%) e, em pequena quantidade, de lignina (3 - 14\%). Entre os nutrientes avaliados, houve liberação de $\mathrm{N}(6-19 \%), \mathrm{K}(49-60 \%), \mathrm{Ca}(18-20 \%)$ e $\mathrm{Mg}(27-39 \%)$, enquanto o P foi acumulado (-20 e $-40 \%)$. Na região norte fluminense, a dinâmica da decomposição e liberação de nutrientes da serapilheira foliar de eucalipto, durante 382 dias, evidenciou a liberação de $\mathrm{K}>\mathrm{Mg}>\mathrm{Ca}>\mathrm{N}$ e a imobilização de $\mathrm{P}$, sendo marcadamente influenciada pela precipitação pluvial.
\end{abstract}

Palavras-chave: Ciclagem de nutrientes, celulose, lignina, polifenóis e eucalipto.

\section{DECOMPOSITION AND NUTRIENT RELEASE FROM LEAF LITTER OF Eucalyptus grandis IN PLANTATIONS AND REGROWTH SISTEMS IN NORTHERN RIO DE JANEIRO STATE, BRAZIL}

\begin{abstract}
This work aimed to evaluate the decomposition and nutrient release of leaf litter in 8-year old Eucalyptus grandis first rotation and 2 and 5-year old regrowth systems through litter bag technique. The annual average temperature was $19.5^{\circ} \mathrm{C}$ and annual rainfall was $848 \mathrm{~mm}$, with $54 \%$ of rains concentrated in the period from October to December. The results showed that the dry weight loss, nutrient release ( $N$, $K, \mathrm{Ca}$ and $\mathrm{Mg}$ ) and organic fraction release (lignin, cellulose and polyphenols) were similar for the first rotation and regrowth systems. The mass loss percentage, mean of three sites, fitted a quadratic model
\end{abstract}

\footnotetext{
${ }^{1}$ Recebido em 16.12.2003 e aceito para publicação em 20.04.2005.

${ }^{2}$ Instituto Superior de Ciências Agrárias FAETEC-RJ. E-mail: <scgilmar@bol.com.br>.

${ }^{3}$ Laboratório de Solos do Centro de Ciências e Tecnologias Agropecuárias (CCTA) da Universidade Estadual do Norte Fluminense (UENF). E-mail: <tonygama@uenf.br>.

${ }^{4}$ Programa de Pós-Graduação em Produção Vegetal da Universidade Federal do Espírito Santo - UFES.
} 
$\left(\mathrm{y}=99.443+0.0348 \mathrm{x}-0.0003 \mathrm{x}^{2} ; \mathrm{R}^{2}=0.92 ; \mathrm{p}=0.0225\right)$. Only in the rainfall period, effective mass loss $(29$ $-33 \%)$, cellulose release ( $40-51 \%)$, polyphenols $(54-74 \%)$ and low lignin release $(3-14 \%)$ was observed. Among the nutrients, release of N (6-19\%), K (49-60\%), Ca (18-20\%), Mg (39-27\%) and immobilization of $P(-20$ and $-40 \%)$ was observed. In northern Rio de Janeiro State, the eucalypt leaf litter decomposition dynamics showed release of $K>M g>C a>N$, over 382 days, and immobilization of $P$, markedly influenced by rainfall.

Keywords: Nutrient cycling, cellulose, lignin, polyphenols and eucalypt.

\section{INTRODUÇÃO}

Em sistemas florestais, a compreensão dos fatores que regulam a decomposição pode assumir importante papel no manejo de plantios florestais, possibilitando a elaboração de técnicas de cultivo que melhorem a utilização de nutrientes contidos nos resíduos vegetais que formam a serapilheira. A decomposição é regulada pela interação de três grupos de variáveis: as condições físico-químicas do ambiente, as quais são controladas pelo clima e pelas características edáficas do sítio; a qualidade (orgânica e nutricional) do substrato, que determina sua degradabilidade; e a natureza da comunidade decompositora, os macro e microrganismos (HEAL et al., 1997; CORREIAe ANDRADE, 1999). De modo geral, o clima controla o processo de decomposição em escala regional, enquanto a composição química domina o processo em escala local (BERG, 2000).

Em plantios de Eucalyptus sp são relatadas baixas taxas de decomposição, acarretando acúmulo de material orgânico e, por conseguinte, aumento da quantidade de nutrientes na interface serapilheira-solo (ADAMS e ATTIWIL, 1986; LOUZADA et al., 1997; GAMARODRIGUES e BARROS, 2002). Essa baixa taxa de decomposição seria, em parte, decorrente da eficiente retranslocação de nutrientes (ciclagem bioquímica) pelo eucalipto, produzindo serapilheira de baixa qualidade nutricional, especialmente em $\mathrm{NeP}$ (GAMA-RODRIGUES e BARROS, 2002). Isso acarretaria, ao menos no primeiro ano de decomposição, a predominância do processo de imobilização desses elementos (GUO e SIMS, 1999; GAMA-RODRIGUES e BARROS, 2002).

Desse modo, o desenvolvimento de práticas de manejo em sistemas florestais deve considerar a ciclagem de nutrientes como um componente da produtividade, sendo a velocidade de decomposição da serapilheira um mecanismo de controle desse processo (ADAMS e ATTIWIL, 1986). Dessa forma, o entendimento da liberação de nutrientes da serapilheira em sincronia com as necessidades das plantas, associado às variações sazonais do clima, poderia contribuir para a formulação de modelos para manejo sustentável de plantios florestais. O objetivo deste trabalho foi avaliar a decomposição e a liberação de nutrientes da serapilheira foliar, em povoamentos de Eucalyptus grandis, formados por um plantio de primeira rotação, com 8 anos, e duas rebrotas, com 2 e 5 anos de idade, na região norte fluminense.

\section{MATERIAL E MÉTODOS}

O trabalho foi conduzido no município de São Francisco do Itabapoana, na região norte do Estado do Rio de Janeiro, Brasil. A área experimental situavase em um Argissolo Amarelo, ácido e de baixa fertilidade (Quadro 1), na região dos tabuleiros costeiros. No período experimental, a temperatura média anual foi de $22,7^{\circ} \mathrm{C}$, sendo a mínima de $19,5^{\circ} \mathrm{C}$ e a máxima, de $26,7^{\circ} \mathrm{C}$, enquanto a precipitação pluvial foi de $848 \mathrm{~mm}$, ocorrendo $54 \%$ das chuvas no período de outubro a dezembro (Figura 1).

Quadro 1 - Características químicas do solo, na profundidade de $0-20 \mathrm{~cm}$, sob povoamentos de E. grandis na região norte fluminense

Table 1 - Soil chemical characteristics, in the deep 0-20 $\mathrm{cm}$, under plantations E. grandis, in North Rio de Janeiro - Brazil

\begin{tabular}{|c|c|c|c|c|c|c|c|}
\hline *Pov. & $\mathrm{pH}$ & $\mathrm{K}^{+}$ & $\mathrm{Ca}^{2+}$ & $\mathrm{Mg}^{2+}$ & $\mathrm{Al}^{3+}$ & $\mathrm{H}+\mathrm{Al}$ & $\mathrm{P}$ \\
\hline & & \multicolumn{5}{|c|}{$\mathrm{mmol}_{\mathrm{c}} \mathrm{dm}^{-3}$} & $\mathrm{mg} \mathrm{dm}^{-3}$ \\
\hline PGR8 & 4,88 & 0,9 & 6,3 & 3,2 & 4,5 & 38,0 & 3,1 \\
\hline RGR2 & 4,82 & 0,6 & 7,3 & 2,9 & 3,6 & 42,9 & 2,8 \\
\hline RGR5 & 4,89 & 0,6 & 6,2 & 3,5 & 3,6 & 36,2 & 2,7 \\
\hline
\end{tabular}

*Pov. - povoamentos; PGR8 - Plantio de E. grandis, 8 anos; RGR2 - Rebrota de E. grandis, 2 anos;e RGR5 - Rebrota de E. grandis, 5 anos. 


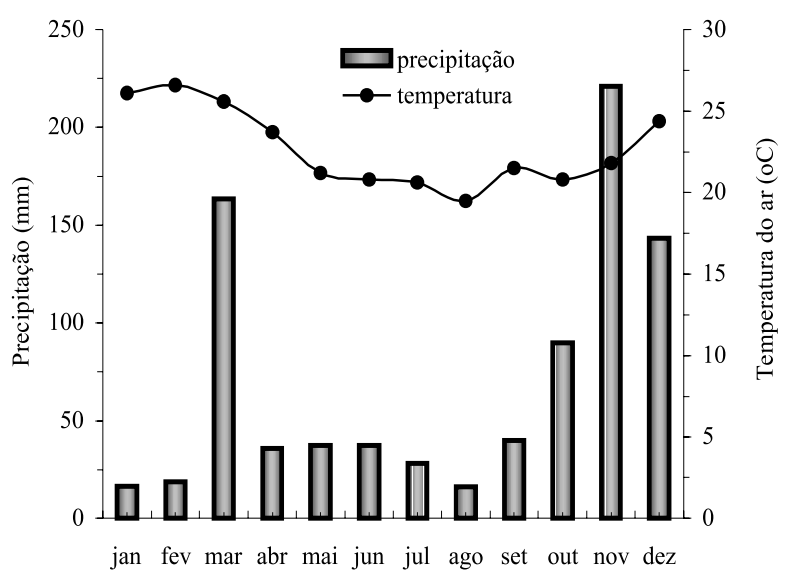

Figura 1 - Precipitação pluvial e temperatura do ar, durante o período de estudo de decomposição da serapilheira foliar de E. grandis, em São Francisco de Itabapoana, norte do Rio de Janeiro.

Figure 1-Rainfall and air temperature over the study period of foliar litter decomposition E. grandis in São Francisco do Itabopoana, Rio de Janeiro State -Brazil.

Os povoamentos avaliados foram de Eucalyptus grandis, distintos quanto à idade do plantio e manejo, classificados da seguinte forma: PGR8, plantio com 8 anos de idade; RGR2, rebrota com 2 anos de idade; e RGR5, rebrota com 5 anos de idade. Uma porção do folhedo recém-caído foi coletada e colocada em sacos de decomposição de $1 \mathrm{~mm}$ de malha (náilon), com dimensões de 20 × $20 \mathrm{~cm}$ (ANDERSON e INGRAM, 1996), deixando-se espaços que permitiam a entrada da meso e macrofauna. A massa seca referente ao tempo inicial (Ti) foi obtida mediante um fator de correção após a secagem em estufa a $75^{\circ} \mathrm{C}$ até peso constante. Em cada povoamento florestal, prepararam-se 20 sacos de decomposição, que foram colocados sobre a serapilheira acumulada. Durante o período experimental, iniciado em janeiro de 1999, foram coletados quatro sacos, por época de amostragem, ou seja, aos 60, 120, 180,270 e 382 dias. Estes foram limpos com o auxílio de um pincel, retirando-se o solo aderido, sendo o material remanescente secado e pesado.

Nas amostras, determinaram-se os teores de carbono, pela digestão de $100 \mathrm{mg}$ de material vegetal

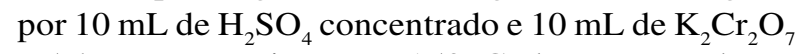
a $5 \%$, com aquecimento a $150{ }^{\circ} \mathrm{C}$, durante uma hora, utilizando uma curva-padrão de C (sacarose) e a leitura da absorbância a 600 nm (ANDERSON e INGRAM, 1996); as concentrações de polifenóis solúveis totais foram obtidas através da extração de uma alíquota de $200 \mathrm{mg}$ de material vegetal em $50 \mathrm{~mL}$ em metanol 50\%, na temperatura controlada entre $77-80^{\circ} \mathrm{C}$, durante uma hora, utilizando como padrão o ácido tânico e, para o desenvolvimento de cor, o reagente de Folin-Denis, realizando-se a leitura de absorbância a $760 \mathrm{~nm}$ (ANDERSON e INGRAM, 1996). Na determinação de celulose e lignina, empregou-se o método de fibra em detergente ácido (FDA), baseado no método de Van Soest e Wine (1968), com adaptações propostas por Anderson e Ingram (1996). O N foi determinado em um analisador automático, utilizando-se a digestão Kjeldahl (BREMMER e MULVANEY, 1982). Em uma alíquota do material vegetal utilizou-se a digestão nítricoperclórica, e do extrato determinou-se o $\mathrm{P}$ por colorimetria, sendo K lido em fotômetro de chama e o Ca e Mg, em espectrofotômetro de absorção atômica (BATAGLIA et al., 1983).

A matéria seca decomposta em função do tempo foi calculada pela diferença entre a massa inicial (12 g) e a massa determinada ao final de cada amostragem, obtendo-se, assim, o porcentual de matéria seca remanescente. Com esses dados, estimaram-se as taxas da decomposição $(k)$ por diversos modelos de regressão comumente usados em estudos de decomposição (WIEDER e LANG, 1982). O mesmo procedimento foi empregado para o porcentual de massa remanescente de lignina, celulose, polifenóis e nutrientes. Na análise de variância dos dados de perdas de massa e da composição química, adotou-se o delineamento inteiramente casualizado, de acordo com Wieder e Lang (1982).

\section{RESULTADOS E DISCUSSÃO}

A análise de variância revelou ausência de diferenças significativas entre os povoamentos de eucalipto quanto à perda de massa e às demais variáveis avaliadas. Em razão disso, foi ajustada uma única regressão dos três povoamentos. Em perda de massa, o melhor ajuste foi o modelo quadrático (Figura 2), que, de acordo com Wieder e Lang (1982), pode descrever a variação da perda de massa compreendida em um período específico de amostragem. No presente trabalho, o modelo quadrático indicou a forte influência da precipitação pluviométrica sobre as taxas de decomposição, evidenciando-se maior perda de massa 
no período de maior concentração das chuvas (Figuras 1 e 2). A perda de massa mostrou-se altamente correlacionada com a precipitação acumulada $(r=0,98$; $\mathrm{p}<0,01$ ), indicando que o padrão climático regional atuaria como principal fator regulador do processo de decomposição.

No final do período de decomposição, a perda de massa foi de aproximadamente $30 \%$. Esse valor foi similar ao observado por Guo e Sims (1999). Geralmente, as florestas de eucalipto apresentam baixas taxas de decomposição, normalmente inferiores a 50\% durante o ano, sob diferentes sistemas de manejo e condições edafoclimáticas (ADAMS e ATTIWILL, 1986; LOUZADA etal., 1997; GAMA-RODRIGUESe BARROS, 2002).

A quantidade de lignina aumentou nos três povoamentos até os 286 dias (Figura 3). Mas, após 382 dias, houve liberação de lignina de aproximadamente $5 \%$ no PGR8 e de $10 \%$ no RGR2 e RGR5. Aumentos iniciais do conteúdo de lignina foram também verificados por Musvoto et al. (2000), em estudo sobre a decomposição de folhas de mangueira, o que foi associado à entrada de pequenas partículas orgânicas nos sacos de decomposição. Essas partículas em estádios mais avançados de decomposição seriam pobres em compostos orgânicos solúveis, mas ricas em compostos

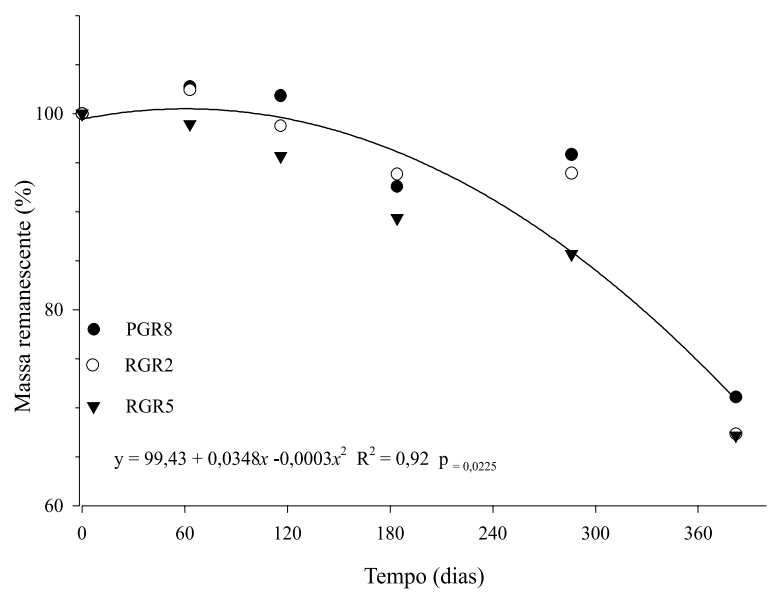

Figura 2 - Matéria seca remanescente da serapilheira foliar de E. grandis no período de 382 dias. PGR -8 anos, RGR2 - 2 anos e RGR5 - 5 anos.

Figure 2-Dry matter remaining over a 382-day period of E. grandis foliar litter. $P G R-8$ years; RGR22 years; $R G R 5$ - 5 years.

R. Árvore, Viçosa-MG, v.29, n.4, p.563-570, 2005 recalcitrantes (MUSVOTO et al., 2000). Houve aumento da concentração de lignina no material remanescente final dos três povoamentos avaliados (Quadro 2). Essa fração orgânica é descrita como a mais resistente à degradação (HAMMEL, 1997), o que proporcionaria aumento da sua concentração ao longo do processo de decomposição, em função da liberação de formas mais solúveis de carbono.

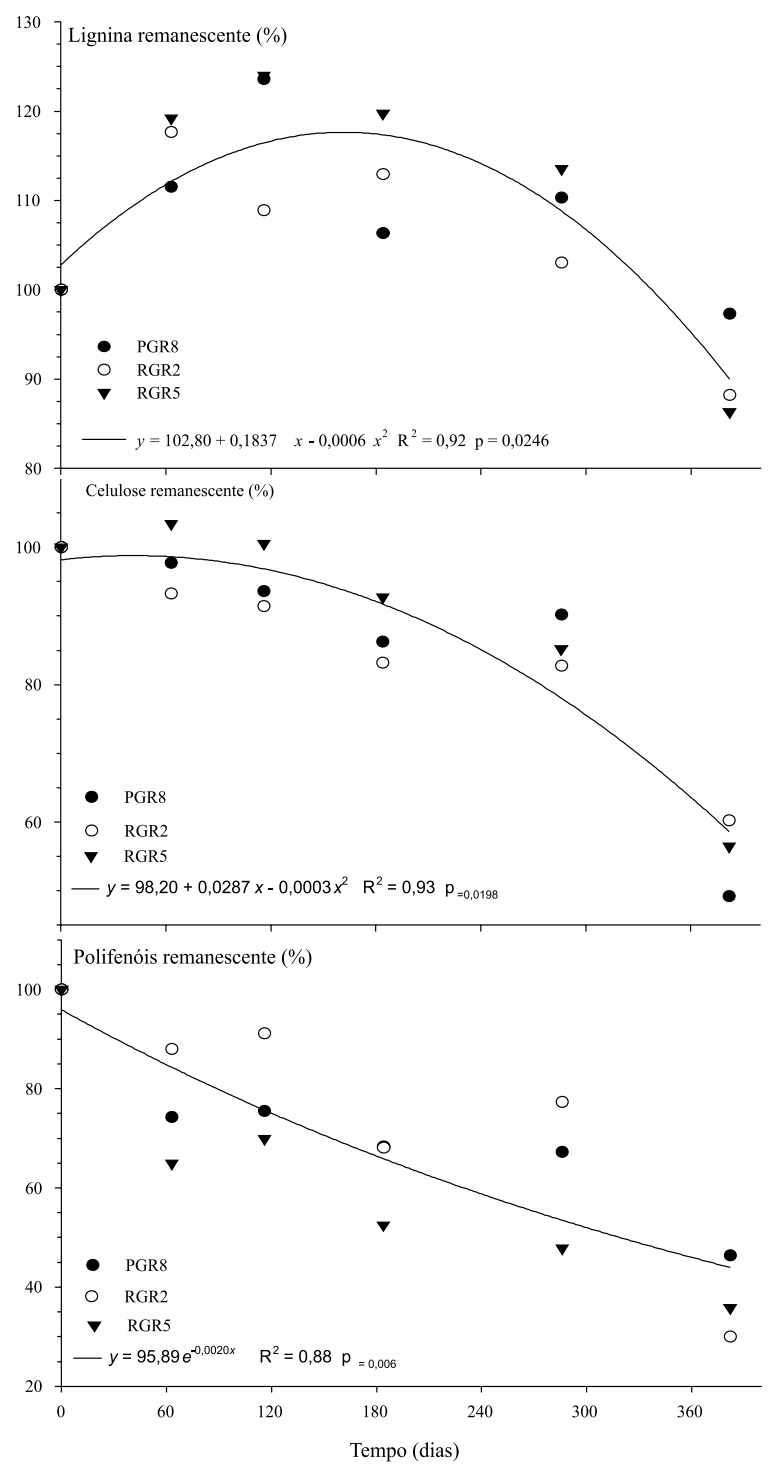

Figura 3-Lignina, celulose e polifenóis remanescentes na serapilheira foliar de $E$. grandis no período de 382 dias. PGR 8 anos, RGR 2 - 2 anos e RGR5 - 5 anos.

Figure 3 - Remaining lignin, cellulose and polyphenols of E. grandis foliar litter over 382-day period. PGR -8 years; RGR 2 - 2 years; $R G R 5$ - 5 years. 
Quadro 2 - Composição química de serapilheira foliar no início e final do período de decomposição em povoamentos de E. grandis

Table 2-Chemical composition of foliar litter at the begining and end of the decomposition period in of $E$. grandis plantations

\begin{tabular}{|c|c|c|c|c|}
\hline & Estádio & PGR8** & RGR2 & RGR5 \\
\hline & \multicolumn{4}{|c|}{$\mathrm{g} \mathrm{kg}^{-1}$} \\
\hline \multirow[t]{2}{*}{$\mathrm{C}$} & $\mathrm{I} *$ & 479 & 473 & 490 \\
\hline & $\mathrm{F}$ & 493 & 378 & 390 \\
\hline \multirow[t]{2}{*}{$\mathrm{N}$} & I & 12,53 & 9,89 & 9,99 \\
\hline & $\mathrm{F}$ & 13,85 & 11,56 & 11,55 \\
\hline \multirow[t]{2}{*}{$\mathrm{P}$} & I & 0,35 & 0,31 & 0,32 \\
\hline & $\mathrm{F}$ & 0,70 & 0,52 & 0,92 \\
\hline \multirow[t]{2}{*}{$\mathrm{K}$} & I & 1,06 & 1,18 & 1,26 \\
\hline & $\mathrm{F}$ & 0,72 & 0,74 & 0,86 \\
\hline \multirow[t]{2}{*}{$\mathrm{Ca}$} & I & 10,23 & 10,12 & 10,51 \\
\hline & $\mathrm{F}$ & 12,1 & 12,03 & 12,54 \\
\hline \multirow[t]{2}{*}{$\mathrm{Mg}$} & I & 2,42 & 2,16 & 2,70 \\
\hline & $\mathrm{F}$ & 2,16 & 2,55 & 2,66 \\
\hline \multirow[t]{2}{*}{ Lignina } & I & 216 & 205 & 197 \\
\hline & $\mathrm{F}$ & 283 & 233 & 254 \\
\hline \multirow[t]{2}{*}{ Celulose } & I & 184 & 188 & 171 \\
\hline & $\mathrm{F}$ & 142 & 168 & 156 \\
\hline \multirow[t]{2}{*}{ Polifenóis } & I & 61 & 57 & 60 \\
\hline & $\mathrm{F}$ & 17 & 23 & 30 \\
\hline
\end{tabular}

* $\mathrm{I}=$ inicial e $\mathrm{F}=$ final.

** PGR8 - Plantio de E. grandis, 8 anos; RGR2 - Rebrota de $E$. grandis, 2 anos; e RGR5 - Rebrota de $E$. grandis, 5 anos.

A liberação de celulose foi pequena no período de menores precipitações pluviométricas (Figura 1), mas com acentuado aumento na estação chuvosa, sendo no final do período de decomposição de, aproximadamente $55 \%$ (Figura 3). A serapilheira foliar dos três povoamentos mostrou elevados teores iniciais de celulose, que variou de $171-188 \mathrm{~g} \mathrm{~kg}^{-1}$, indicando baixo potencial de decomposição (Quadro 2). De acordo com Guo e Sims (2002), os teores iniciais de celulose eaperda de massa da serapilheira foliar exibiram altocoeficiente de correlação negativa: $150,2 \mathrm{~g} \mathrm{~kg}^{-1}$ no $E$. bortryoides, 105,0 $\mathrm{g} \mathrm{kg}^{-1}$ no E. globulus e $179,4 \mathrm{~g} \mathrm{~kg}^{-1}$ no E. ovata. Houve redução da concentração de celulose ao final do período de decomposição (Quadro 2), provavelmente associada à liberação da fração não ligada a lignina e a hemicelulose na parede celular.

As elevadas concentrações iniciais de lignina e celulose estão relacionadas com a espécie estudada (GAMA-RODRIGUES e BARROS, 2002; GUO e SIMS, 2002), assim como a senescência do material foliar, proporcionando a retranslocação de frações solúveis e permanência desses compostos recalcitrantes (HAMMEL, 1997). A presença dessas frações orgânicas conferiria resistência ao material vegetal, dificultando a penetração de organismos decompositores por meio da parede celular (GALLARDO e MERINO, 1993), contribuindo para a baixa velocidade de decomposição.

A serapilheira de eucalipto apresentou elevada concentração inicial de polifenóis variando de $57 \mathrm{~g}$ $\mathrm{kg}^{-1}$ a $61 \mathrm{~g} \mathrm{~kg}^{-1}$ (Quadro 2). A dinâmica de liberação dessa fração orgânica ajustou-se ao modelo exponencial simples (Figura 3), ao contrário do observado na lignina e na celulose. Por meio desse modelo, estimou-se em $45 \%$ a quantidade de polifenóis remanescentes, ou seja, no final do período de decomposição houve a liberação de $55 \%$ desses compostos, resultando na redução do teor médio final para $23 \mathrm{~g} \mathrm{~kg}^{-1}$ (Quadro 2). A atuação de polifenóis no processo de decomposição estaria relacionada com a complexação de $\mathrm{N}$, diminuindo sua liberação da serapilheira (CONSTANTINIDES e FOWNES, 1994; HANDAYANTO et al., 1997). Palm et al. (2001) relataram que materiais com teores superiores a $40 \mathrm{~g} \mathrm{~kg}^{-1}$ de polifenóis limitariam a liberação de $\mathrm{N}$.

Essa liberação oscilou entre pequenas liberações e imobilizações, ocorrendo a efetiva liberação (10 a $19 \%$ ) durante o período de maiores precipitações pluviométricas (Figuras 1 e 4). Entretanto, ocorreu acúmulo de $\mathrm{P}$, alcançando valor máximo aos 286 dias, sem que houvesse liberação líquida no final do período de decomposição (Figura 4). O nível de acumulação de $\mathrm{P}$ seria um indicativo de que este foi o nutriente mais limitante do processo de decomposição nos plantios de eucalipto avaliados. A eficiência da ciclagem bioquímica (GAMA-RODRIGUES e BARROS, 2002), associada aos baixos teores de P no solo (Quadro 1), foi um fator que proporcionou a serapilheira foliar a apresentar, inicialmente, a relação C/P acima de 1300 . Essa relação, ao final do período de decomposição, encontrava-se em torno de 450, ainda superior ao indicado para que ocorra a mineralização (STEVENSON, 1986).

A liberação de $\mathrm{N}$ e a acumulação de $\mathrm{P}$ foram menores que as observadas por Gama-Rodrigues e Barros (2002), em Eucalyptus grandis e E. urophyla, no sul da Bahia, onde as precipitações foram maiores e mais bem distribuídas durante o ano. No entanto, Guo e Sims (1999), em plantios de E. brookerana e E. botryoides, na Nova Zelândia, observaram acumulação de N, sendo a de $\mathrm{P}$ similar à do presente trabalho. Vários estudos

R. Árvore, Viçosa-MG, v.29, n.4, p.563-570, 2005 
indicaram que as folhas de eucalipto são decompostas lentamente, resultando na acumulação e formação de um estoque significativo de nutrientes, especialmente de $\mathrm{P}$ e N, na serapilheira acumulada sobre a superfície (ADAMSeATWILL, 1986;O`CONNELL, 1988;LOUZADA et al., 1997; GAMA-RODRIGUES e BARROS, 2002).

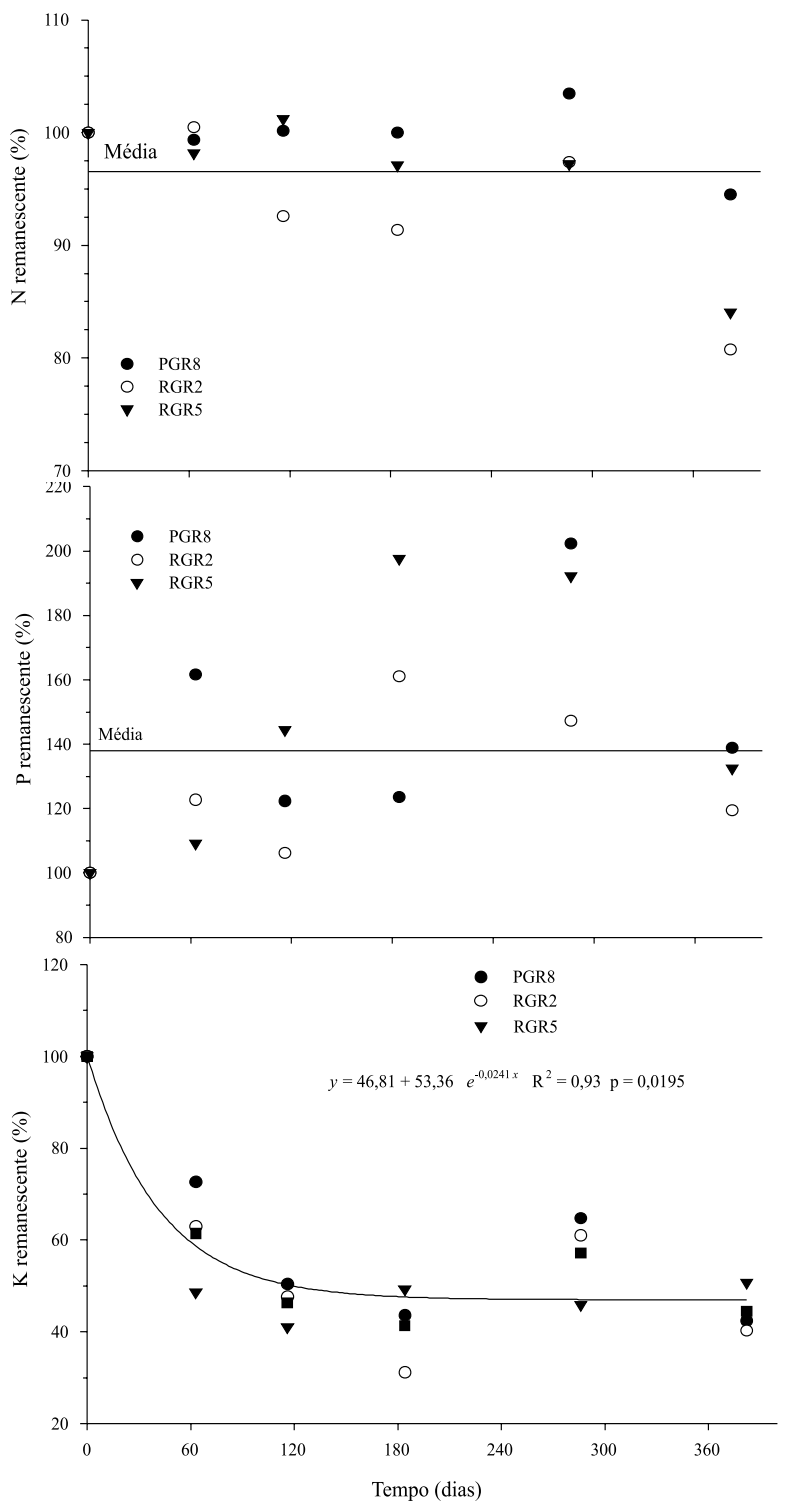

Figura 4 - Nitrogênio, fósforo e potássio remanescentes na serapilheira foliar de $E$. grandis. PGR -8 anos, RGR2 - 2 anos e RGR5 - 5 anos.

Figure 4-Remaining nitrogen, phosphorus and potassium of E. grandis foliar litter over a 382-day period. $P G R-8$ years; $R G R 2-2$ years; $R G R 5-5$ years.

R. Árvore, Viçosa-MG, v.29, n.4, p.563-570, 2005
O K foi rapidamente liberado da serapilheira, ou seja, em torno de $50 \%$ até os 116 dias. Após esse período, o K remanescente manteve-se constante até o final do período de decomposição, conforme o modelo exponencial ajustado (Figura 4). A lixiviação seria um dos principais mecanismos de transferência do elemento para o solo, uma vez que ele não é componente estrutural de qualquer composto das plantas e a mineralização não seria um pré-requisito para sua liberação(O'CONNELL, 1988; GAMARODRIGUES E BARROS, 2002).

OCa oscilou entre ligeira liberação $(7 \%)$ e acumulação (5\%) até os 286 dias, mas, ao final do período de decomposição, houve liberação líquida entre 18 e $20 \%$ (Figura 5). Gama- Rodrigues e Barros (2002) relataram liberação de Ca em torno de $26 \%$, enquanto Guo e Sims (2002) evidenciaram acúmulo de Ca ao final de 12 meses de decomposição. O Mg apresentou liberação líquida de $33 \%$, de acordo com o modelo exponencial ajustado (Figura 5).

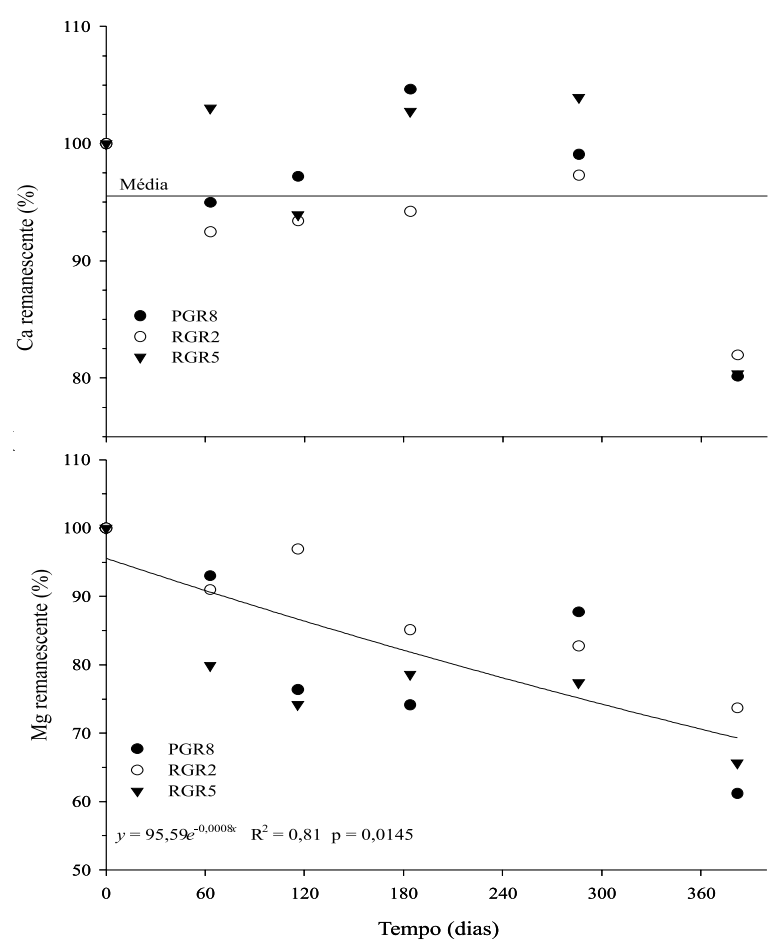

Figura 5 - Cálcio e magnésio remanescente na serapilheira foliar em povoamentos de $E$. grandis na região norte fluminense. PGR -8 anos, RGR2 -2 anos e RGR5 - 5 anos.

Figure 5 - Remaining calcium and magnesium of E. grandis foliar litter over a 382-day period. $P G R-8$ years; $R G R 2$ - 2 years; RGR 5 - 5 years. 
No Quadro 3 são mostradas as correlações entre as variáveis analisadas. Observa-se, nesse quadro, que as liberações de $\mathrm{Ne} \mathrm{Mg}$ e das frações orgânicas estão associadas positivamente à perda de massa. Correlações positivas também ocorreram entre as frações orgânicas e dessas com $\mathrm{N}$ e Mg. A liberação de N correlacionou-se com as de Cae Mg. Segundo Gama-RodrigueseBarros (2002), aliberaçãodeMgacompanharia a perda de massa.

Quadro 3 - Coeficientes de correlação entre o porcentual de matéria seca remanescente, frações orgânicas (celulose, lignina e polifenóis) e nutrientes ( $, \mathrm{P}, \mathrm{K}, \mathrm{Ca}$ e $\mathrm{Mg}$ ) da serapilheira foliar em plantios de $E$. grandis no período de 382 dias

Table 3 - Correlation coefficients between percent of dry weight remaining, organic fractions (cellulose, lignin and polyphenol) and nutrients $(N, P, K, C a$ and $M g)$ of foliar litter at plantations of E. grandis over 382 days

\begin{tabular}{|c|c|c|c|c|c|c|c|c|c|}
\hline & MR & Cel. & Lig. & Pol. & $\mathrm{N}$ & $\mathrm{P}$ & $\mathrm{K}$ & $\mathrm{Ca}$ & $\mathrm{Mg}$ \\
\hline MR & 1,00 & $0,99 * *$ & $0,98 * *$ & $0,98 * *$ & $0,95^{*}$ & 0,06 & 0,47 & 0,82 & $0,97 *$ \\
\hline Cel. & & 1,00 & $0,98 * *$ & $0,98 * *$ & $0,95^{*}$ & 0,07 & 0,45 & 0,84 & $0,97 *$ \\
\hline Lig. & & & 1,00 & $0,98 * *$ & $0,91 *$ & 0,03 & 0,29 & 0,82 & $0,91 *$ \\
\hline Pol. & & & & 1,00 & $0,91 *$ & $-0,06$ & 0,42 & 0,74 & $0,93 *$ \\
\hline $\mathrm{N}$ & & & & & 1,00 & 0,33 & 0,57 & $0,91 *$ & $0,97 * *$ \\
\hline $\mathrm{P}$ & & & & & & 1,00 & 0,14 & 0,58 & 0,15 \\
\hline $\mathrm{K}$ & & & & & & & 1,00 & 0,32 & 0,66 \\
\hline $\mathrm{Ca}$ & & & & & & & & 1,00 & 0,82 \\
\hline $\mathrm{Mg}$ & & & & & & & & & 1,00 \\
\hline
\end{tabular}

Coeficientes de correlação de Pearson, em que $* *(p<0,01)$ e *(p<0,05), sendo MR - massa remanescente; Cel. - celulose; Lig. - lignina; e Pol. - polifenóis.

\section{CONCLUSÕES}

1. A perda de massa e a liberação de frações orgânicas e de nutrientes da serapilheira foliar foram similares no plantio de primeira rotação e nas rebrotas de $E$. grandis.

2. A precipitação pluvial influenciou marcadamente a decomposição da serapilheira foliar.

3. A serapilheira funcionou como fonte (mineralização) de $\mathrm{K}, \mathrm{Mg}$, Ca e $\mathrm{N}$ e como um forte dreno (imobilização) de P.

\section{REFERÊNCIAS BIBLIOGRÁFICAS}

ADAMS, M. A.; ATTIWILL, P.M. Nutrient cycling an nitrogen mineralization in eucalypt forests south-easthern Australia. I. Nutrient Cycling and nitrogen turnover. Plant and Soil, v.92, p.319339, 1986.

ANDERSON, J.D.; INGRAM, J.S.I. Tropical soil biology and fertility: A handbook of methods. 2. ed. Wallingford: CAB International, 1996. $171 \mathrm{p}$.
BATAGLIA, O.C. et al. Métodos de análise química de plantas. Campinas: Instituto Agronômico de Campinas, 1983. 48p. (Boletim Técnico, 78).

BERG, B.. Litter decomposition and organic matter turnover in northern forest soil. Forestry

Ecology Management, v.133, p.13-22, 2000.

BREMNER, J.M.; MULVANEY, C.S. Nitrogen total. In: PAGE, A. L. (Ed.). Methods of soil analysis. 2. ed. Madison: Soil Science Society of America, 1982. v.2. p.595-624.

CONSTANTINIDES, M.; FOWNES, J.H. Nitrogen mineralization from leaves and litter of tropical plants: relationship to nitrogen, lignin and soluble polyphenol concentrations. Soil Biology and Biochemistry, v.26, p.49-55, 1994.

CORREIA, M.E.F.; ANDRADE, A.G. Formação de serapilheira e ciclagem de nutrientes. In: (Eds.). SANTOS, G.A.; CAMARGO, F.A.O.

Fundamentos da Matéria Orgânica do Solo: ecossistemas tropicais e subtropicais. Porto Alegre: Genesis, 1999. p.197-226.

R. Árvore, Viçosa-MG, v.29, n.4, p.563-570, 2005 
GAMA-RODRIGUES, A.C.; BARROS, N.F.

Ciclagem de nutrientes em floresta natural e em plantios de eucalipto e de dandá no sudeste da Bahia, Brasil. Revista Árvore, v.26, n.2, p.193-207, 2002.

GALLARDO, A.; MERINO, J. Leaf decomposition in two Mediterranean ecossystems of Southeast Spain: influence substrate quality. Ecology, v.74, p.721-727, 1993.

GUO, L.B.; SIMS, R.E.H. Litter decomposition and nutrient release via litter decomposition in the New Zealand eucalypt short rotation forests.

Agriculture Ecosystems and

Environment, v.75, p.133-140, 1999.

GUO, L.B.; SIMS, R.E.H. Eucalypt litter decomposition and nutrient release under a short rotation forest regime and effluent irrigation treatments in New Zealand: II. Internal effects. Soil Biology and Biochemistry, v.34, p.913-922, 2002.

HAMMEL, K.E. Fungal degradation of lignin. In CADISH, G.; GILLER, K.E., (Eds.) Driven by Nature: plant litter quality and decomposition. Walingford: CAB International, 1997. 33-46p.

HANDAYANTO, E.; GILLER, K.E.; CADISH, G. Regulating $\mathrm{N}$ release from legume tree prunings by mixing residues of different quality. Soil Biology and Biochemistry, v.29, p.14171426, 1997.

HEAL, O.W.; ANDERSON J.M.; SWIFT, M.J. Plant litter quality and decomposition: an historical overview. P 3-30. In: CADISH, G.; GILLER, K.E. (Eds.). Driven by Nature: plant litter quality and decomposition. Walingford: CAB International, 1997. $409 \mathrm{p}$.
LOUZADA, J.N.C. et al. Litter decomposition in semidecidous forest and Eucalyptus spp. crop in Brazil: a comparison. Forest Ecology and Management, v.94, p.31-36,1997.

MUSVOTO, C.; CAMPBELL, B.M.; KIRCHMANN, $\mathrm{H}$. Decomposition and nutrient release from mango and miombo woodland litter in Zimbabwe. Soil Biology and Biochemistry, v.32, p.1111-1119, 2000.

NOVAIS, R.F.; SMITH, T.J. Fósforo em solo e planta em condições tropicais. Viçosa: UFV/DPS, 1999. 399 p.

O'CONNELL, A.M. Nutrient dynamics in decomposing litter in karri (Eucalyptus diversicolor $\mathrm{F}$. Muell.) forests of South Western Australia. Journal of Ecology, v. 76, p. 11861203, 1988.

PALM, C.A. et al. Organic inputs for soil fertility management in tropical agroecosystems: application of an organic resource database. Agriculture Ecosystems Environment, v.83, n.1-2, p.27-42, 2001.

STEVENSON, F.J. Cycles of soil carbon, nitrogen, phosphorus, sulphur, micronutrients. New York: Wiley \& Sons, 1986.380p.

Van SOEST, P.; WINE, R.H. Development of a comprehensive system of feed analysis and applications to forages. Journal of Associate Official Agronomy Chemistry, v.51, p.780-785, 1968 .

WIEDER, R.K.; LANG, G.E. A critique of the analytical methods used examining decomposition data obtained from litter bags. Ecology, v.63, p.1636-1642, 1982. 